26(3), 495-509

\title{
On the Application of Multivariate Kendall's Tau and Its Interpretation
}

\author{
Woojoo Lee ${ }^{a} \cdot$ Jae Youn $\mathrm{Ahn}^{b, 1}$ \\ ${ }^{a}$ Department of Statistics, Inha University; ${ }^{b}$ Department of Statistics, Ewha Womans University
}

(Received April 2, 2013; Revised April 28, 2013; Accepted May 15, 2013)

\begin{abstract}
We study multivariate extension of Kendall's tau and its statistical interpretation. There exist various versions of multivariate Kendall's tau, for example Scarsini (1984), Joe (1990) and Genest et al. (2011); however, few of them mention its lower bounds. For the bivariate case, the Fréchet-Hoeffding lower bound can achieve the lower bound of Kendall's tau. However in the multivariate case, the Fréchet-Hoeffding lower bound itself does not exist as a distribution, which makes the interpretation of Kendall's tau unclear when it has negative value. In this paper, we explain sufficient conditions to achieve the lower bound of Kendall's tau and provide real data examples that provide further insights into the interpretation for the lower bounds of Kendall's tau.
\end{abstract}

Keywords: Copula, Kendall's tau, Comonotonicity, Fréchet-Hoeffding lower bound.

\section{1. 서론}

두 변수 사이의 관계를 설명해주는 대표적인 측도로서 피어슨의 상관계수(Pearson's correlation)가 있 다. 그러나 피어슨의 상관계수는 두 변수의 선형관계를 살펴보는 것에 국한되어있고, 이상치(outlier)에 의해 그 값이 크게 변할 수 있다는 단점이 있다. 따라서 이러한 단점을 보완해 줄 수 있는 다양한 측도 들이 연구되고 있다. 그 중 많이 연구되는 측도 중의 하나인 캔달의 타우(Kendall's tau)는 두 변수 사 이의 일치성(concordance)에 대한 비모수량으로서, 변수의 단조 증가 변환에 대해 통계량이 변하지 않 는 중요한 성질을 가지고 있어서 이상치에 대한 로버스트 성질을 가지고 있다. 또한, 이변량 프레쳇-호 프딩(Fréchet-Hoeffding) 상한에서 최대값인 1 을, 이변량 프레쳇-호프딩 하한에서 최소값인 -1 을 갖는 다는 것이 잘 알려져 있다 (Joe, 1990). 이러한 성질은 피어슨의 상관계수가 가지고 있지 않은 성질이 고, 특히 결합분포가 정규분포가 아닐때에 피어슨의 상관계수가 갖는 한계를 보완할 수 있다.

켄달의 타우는 본래 이변량 변수에 대해 정의되었으나, 이후 3 차원 이상의 변수에도 적용될 수 있도록 몇몇 확장이 연구되어 왔다 (Genest 등, 2011). 특히 금융 및 보험 분야에서 다차원 자료의 일치성을 측 정하기 위한 도구로 켄달의 타우를 사용하는 경우가 빈번하게 발견된다 (Cherubini 등, 2004). 그러나 다차원의 경우 켄달의 타우 값이 의미하는 바를 즉각적으로 이해하기란 쉽지가 않다. 예를 들어 3 차원

\footnotetext{
This work was supported by Inha University Research Grant (INHA-44770-01).

${ }^{1}$ Corresponding author: Assistant Professor, Department of Statistics, Ewha Womans University, 11-1 Daehyun-Dong, Seodaemun-Gu, Seoul 120-750, Korea. E-mail: jaeyahn@ewha.ac.kr
} 
자료의 경우, 켄달의 타우 통계량 값이 -0.3 으로 관측되었다면 우리는 이것을 어떻게 해석해야 할까? 이변량의 경우에는 프레쳇-호프딩 하한의 특성으로부터 한 변수가 다른 변수의 단조 감소 변환으로 표현 될 수 있는 경우 켄달의 타우 값이 -1 이 되고, 그것이 최소값이 된다는 사실이 알려져 있으므로, 이를 바탕으로 두 변수의 관계를 짐작할 수 있다. 그러나 3 차원 이상의 경우에는 프레쳇-호프딩 하한은 코퓰 라에 대응되지 않으므로 2 차원에서의 결과를 그대로 확장하는 것이 어렵다. 따라서 변수들 사이가 어떠 한 관계일 때 음수값이 나올 수 있는가에 대한 통계적 고찰이 필요하다. 본 논문에서는 다차원 자료에 대한 켄달의 타우의 확장에 대해 살펴보고, 통계량의 값이 상한, 하한일 때의 변수들 사이의 관계에 대 해 고찰을 해본다. 특히, 다차원의 경우 실제 사례를 통해 켄달의 타우 통계량이 음수 값을 갖는 경우 변 수들이 실제 본 논문에서 고찰하고 있는 조건이 만족되고 있는가를 살펴볼 것이다.

본 논문의 순서는 다음과 같다. 2 절에서는 코퓰라(copula)의 기본적인 내용을 소개하고, 3 절에서는 일 치성의 측도로서 켄달의 타우에 대해 기본적인 수학적 성질을 살펴보고 이론적인 배경을 소개한다. 특 히, 3 차원 이상의 문제에서 켄달의 타우를 어떻게 정의하는지 살펴본다. 2 차원의 경우에는 임의의 코퓰 라의 상한과 하한이 잘 알려졌기 때문에 켄달의 타우 값의 상한과 하한에 대해 쉽게 이해할 수 있다. 그 러나 3 차원 이상의 경우에는 그렇지 않다. 따라서 켄달의 타우의 하한을 얻을 수 있는 변수들의 조건으 로서 우리는 $d$-countermonotonicity 개념을 도입하고, 이것이 어떠한 의미를 갖는지 자세히 살펴보도 록 할 것이다. 4 절에서는 $d$-countermonotonicity 조건하에서 켄달의 타우의 이론적이 성질에 대해 살 필 것이며, 5 절에서 실제 자료를 이용하여 분석해 볼 것이다.

\section{2. 코풀라 소개}

본 논문에서는 확률 공간, $(\Omega, \mathcal{F}, P)$, 상의 $d$ 차원 확률벡터를 $\mathbf{X}=\left(X_{1}, X_{2}, \ldots, X_{d}\right)$ 으로 나타내고, $d$ 차 원 실수 벡터를 $\mathbf{x}=\left(x_{1}, x_{2}, \ldots, x_{d}\right)$ 로 나타내기로 한다. 확률벡터 $\mathbf{X}$ 의 결합분포는

$$
F(\mathbf{x})=P\left(X_{1} \leq x_{1}, \ldots, X_{d} \leq x_{d}\right)
$$

으로 정의되고, 각 변수의 주변확률분포는 임의의 실수 $x$ 에 대해 $F_{i}(x)=P\left(X_{i} \leq x\right)$ 으로 나타내 자. 본 논문에서는 주변확률분포들, $F_{i}, i \in\{1, \ldots, d\}$ 이 연속함수임을 가정할 것이다. 집합 $[a, b]^{d}$ 는 $[a, b] \times[a, b] \times \cdots \times[a, b]\left(\subset R^{d}\right)$ 를 뜻하는 것으로 한다. Sklar (1959)에 따르면, 주어진 결합분포 $F$ 에 대해

$$
F(\mathbf{x})=C\left(F_{1}\left(x_{1}\right), \ldots, F_{d}\left(x_{d}\right)\right)
$$

을 만족하는 $C:[0,1]^{d} \mapsto[0,1]$ 가 유일하게 존재한다는 것이 알려졌고, 이 함수를 코퓰라라고 부른다. 코퓰라의 세부적인 내용에 대해서는 Nelson (2006)나 Cherubini 등 (2004)을 참조하면된다.

코퓰라의 여러 가지 특징 가운데에 본 논문과 관련하여 가장 주목할만한 점은 모든 코퓰라는 위, 아래로 유계라는 점이다. $u_{i}=F_{i}\left(x_{i}\right)$ 라 하고, $\mathbf{u}=\left(u_{1}, \ldots, u_{d}\right)$ 라 표시하면, 모든 $\mathbf{u} \in[0,1]^{d}$ 에 대해

$$
\max \left\{u_{1}+\cdots+u_{d}-(d-1), 0\right\} \leq C(\mathbf{u}) \leq \min \left\{u_{1}, \ldots, u_{d}\right\}
$$

이 성립한다. 특별히 부등식의 맨 왼쪽에 있는 함수를 $W(\mathbf{u})$, 오른쪽의 함수를 $M(\mathbf{u})$ 라고 나타내 고 이를 각각 프레쳇-호프딩 하한과 상한이라고 부른다. 여기서 $M(\mathbf{u})$ 은 확률 1 로 $F_{1}\left(X_{1}\right)=\cdots=$ $F_{d}\left(X_{d}\right)$ 이 성립하는 경우 얻어지는 코퓰라이기 때문에 특별히 comonotonic 코풀라라는 이름으로 불리 기도 한다. 이와는 달리 $W(\mathbf{u})$ 는 $d=2$ 인 경우에만 코퓰라가 되는 것으로 밝혀져 있고, Lee와 $\mathrm{Ahn}$ (2013)에서 지적된 바와 같이 3 차원 이상의 코퓰라에 대해서는 하한인 코퓰라가 존재하지 않음이 밝혀 
져 있다. 또한 $X_{1}, \ldots, X_{d}$ 가 독립인 경우, 모든 $\mathbf{u} \in[0,1]^{d}$ 에 대해

$$
\Pi(\mathbf{u})=\prod_{i=1}^{d} u_{i}
$$

으로 나타낼 수 있고, 이는 독립 코퓰라라고 부른다. 또한, 임의의 코퓰라 $C_{1}, C_{2}$ 에 대해 $C_{1} \prec C_{2}$ 는 다 음과 같이 정의한다.

$$
C_{1}(\mathbf{u}) \leq C_{2}(\mathbf{u}) \quad \text { 그리고 } \quad \overline{C_{1}}(\mathbf{u}) \leq \overline{C_{2}}(\mathbf{u}), \quad \text { for all } \mathbf{u} \in[0,1]^{d},
$$

여기서 $\bar{C}$ 는 코퓰라 $C$ 에 대응되는 생존 코퓰라로 정의한다.

\section{3. 켄달의 타우}

먼저 켄달의 타우를 2 차원 상에서 어떻게 정의할 수 있는지 코퓰라의 관점에서 살펴보고, 이것을 다차원 으로 확장한 두 가지 경우를 살펴보기로 하겠다.

\subsection{2 차원 켄달의 타우}

정의 3.1 (켄달의 타우) $\mathbf{X}=\left(X_{1}, X_{2}\right)$ 와 $\mathbf{X}^{*}=\left(X_{1}^{*}, X_{2}^{*}\right)$ 를 이변량 분포 $H(\cdot, \cdot)$ 에서 얻어진 독립인 랜덤벡터라 하자. $\mathbf{X}$ 에 대해 켄달의 타우는 아래와

$$
\tau_{2}(\mathbf{X})=2 \cdot \mathbb{E}\left[\mathbb{I}\left\{\left(X_{1}-X_{1}^{*}\right)\left(X_{2}-X_{2}^{*}\right)>0\right\}\right]-1,
$$

같이 정의된다.

Scarsini (1984)에서는 임의의 2 차원 연속 랜덤벡터 $\mathbf{X}$ 에 대해서 정의되는 실변수 함수, $\kappa$ 가 다음의 공 리 $\mathrm{S}$ 를 만족하게 하면 $\kappa$ 를 일치 측도라고 정의하였다. 편의상 $\kappa(C), \kappa(H), \kappa(X, Y)$ 그리고 $\kappa(\mathbf{X})$ 를 병 행하기로 한다.

\section{공리 $\mathbf{S}$}

S1. $-1 \leq \kappa\left(X_{1}, X_{2}\right) \leq 1, \kappa\left(X_{1}, X_{1}\right)=1$, 그리고 $\kappa\left(X_{1},-X_{1}\right)=-1$;

S2. $\kappa\left(X_{1}, X_{2}\right)=\kappa\left(X_{2}, X_{1}\right)$;

S3. 만약 $X_{1}, X_{2}$ 가 독립이면 $\kappa\left(X_{1}, X_{2}\right)=0$;

S4. $\kappa\left(-X_{1}, X_{2}\right)=\kappa\left(X_{1},-X_{2}\right)=-\kappa\left(X_{1}, X_{2}\right)$;

S5. 만약 두 개의 결합분포 $H_{1}$ 과 $H_{2}$ 가 $H_{1} \prec H_{2}$ 를 만족하면, $\kappa\left(H_{1}\right) \leq \kappa\left(H_{2}\right)$;

S6. 만약 결합분포 $\left\{H_{1}, H_{2}, \ldots\right\}$ 이 $H$ 로 pointwise 수렴하면, $\lim _{n \rightarrow \infty} \kappa\left(H_{n}\right)=\kappa(H)$;

2차원 켄달의 타우가 위의 공리 $\mathrm{S}$ 를 만족 시킴은 알려져 있다 (Nelson, 2006).

\section{2. 다차원 켄달의 타우의 정의와 Concordance Measure}

이제 우리는 다차원 켄달의 타우에 대해서 살펴본다. 켄달의 타우의 $d$ 차원 $(d \geq 2)$ 으로 확장은 다음과 
같이 정의한다.

$$
\begin{aligned}
\tau_{d}(H) & :=c(d)\left[2^{d} \int_{[0,1]^{d}} H(\mathbf{z}) \mathrm{d} H(\mathbf{z})-1\right], \quad \text { with } c(d)=\frac{1}{2^{d-1}-1} \\
T_{d}(H) & :=c(2)\left[2^{2}\left(\begin{array}{l}
d \\
2
\end{array}\right) \sum_{i<j} \int_{[0,1]^{2}}[H]_{i, j}(\mathbf{z}) \mathrm{d}[H]_{i, j}(\mathbf{z})-1\right] \\
& =\left(\begin{array}{l}
d \\
2
\end{array}\right) \sum_{i<j}^{-1}\left[2^{2} \int_{[0,1]^{2}}[H]_{i, j}(\mathbf{z}) \mathrm{d}[H]_{i, j}(\mathbf{z})-1\right],
\end{aligned}
$$

여기서 $[H]_{i, j}$ 는 결합분포 $H$ 의 $i$ 와 $j$ 번째 주변확률변수에 대응하는 2 차원 결합분포로 정의한다. $T_{d}(H)$ 는 Kendall과 Smith (1940)에 의해 처음 정의되었으며, 2 차원에 국한된 Kendall'a tau들의 짝(pairwise) 평균으로 해석될 수 있다. $\tau_{d}(H)$ 는 Joe (1990)에 의해 정의되었고, 식 (3.1)의 다차원으 로의 자연스러운 확장으로, Schmid와 Schmidt (2007)와 비슷한 논법을 사용하여, 다음과 같은 의미에 서 해석될 수 있다. 먼저 2 차원에서의 켄달의 타우를 코퓰라의 관점에서 표현해보자.

$$
\begin{aligned}
\tau_{2}(C) & =4 \int_{[0,1]^{2}} C(\mathbf{u}) \mathrm{d} C(\mathbf{u})-1 \\
= & \frac{\int_{[0,1]^{2}} C(\mathbf{u}) \mathrm{d} C(\mathbf{u})-\int_{[0,1]^{2}} \Pi(\mathbf{u}) \mathrm{d} \Pi(\mathbf{u})}{\int_{[0,1]^{2}} M(\mathbf{u}) \mathrm{d} M(\mathbf{u})-\int_{[0,1]^{2}} \Pi(\mathbf{u}) \mathrm{d} \Pi(\mathbf{u})}
\end{aligned}
$$

이 되는데, 여기서 위의 식 (3.3)의 두 번째 등호는 다음식들

$$
\int_{[0,1]^{2}} M(\mathbf{u}) \mathrm{d} M(\mathbf{u})=\frac{1}{2} \quad \text { 그리고 } \quad \int_{[0,1]^{2}} \Pi(\mathbf{u}) \mathrm{d} \Pi(\mathbf{u})=\left(\frac{1}{2}\right)^{2}
$$

로부터 쉽게 얻어진다. 따라서 $\tau_{2}(C)$ 는 코퓰라 $C$ 와 독립 코퓰라 $\Pi$ 와의 정규화된 거리로 해석될 수 있 고, 다음과 같이 다차원으로 확장될 수 있다.

$$
\begin{aligned}
\tau_{d}(C)=\frac{\int_{[0,1]^{d}} C(\mathbf{u}) \mathrm{d} C(\mathbf{u})-\int_{[0,1]^{d}} \Pi(\mathbf{u}) \mathrm{d} \Pi(\mathbf{u})}{\int_{[0,1]^{d}} M(\mathbf{u}) \mathrm{d} M(\mathbf{u})-\int_{[0,1]^{d}} \Pi(\mathbf{u}) \mathrm{d} \Pi(\mathbf{u})} \\
=\frac{\left(\frac{1}{2}\right)^{d}\left[2^{d} \int_{[0,1]^{d}} C(\mathbf{u}) \mathrm{d} C(\mathbf{u})-1\right]}{\frac{1}{2}-\left(\frac{1}{2}\right)^{d}} \\
=\frac{1}{2^{d-1}-1}\left[2^{d} \int_{[0,1]^{d}} C(\mathbf{u}) \mathrm{d} C(\mathbf{u})-1\right] .
\end{aligned}
$$

위의 식 (3.4)의 두 번째 등호는 다음식들

$$
\int_{[0,1]^{d}} M(\mathbf{u}) \mathrm{d} M(\mathbf{u})=\frac{1}{2} \quad \text { 그리고 } \int_{[0,1]^{d}} \Pi(\mathbf{u}) \mathrm{d} \Pi(\mathbf{u})=\left(\frac{1}{2}\right)^{d}
$$


로부터 쉽게 얻어진다.

Taylor (2007)는 Scarsini (1984) 2차원 일치 측도(measures of concordance) 개념을 아래의 공리 A에 기술된 바와 같이 다차원 일치 측도로 확장하였다. 임의의 $d$ 차원 연속 랜덤벡터 $\mathbf{X}$ 에 대해서 정의되 는 실변수 함수 $\kappa_{d}(\cdot)$ 가 공리 $\mathrm{A}$ 를 만족하면 $\kappa_{d}$ 를 일치 측도라고 정의하였다. 편의상 $\kappa_{d}(C), \kappa_{d}(H)$, $\kappa_{d}\left(X_{1}, \ldots, X_{d}\right)$ 그리고 $\kappa_{d}(\mathbf{X})$ 를 병행하기로 한다.

\section{공리 A}

A1. (Normalization) $\kappa_{p}\left(M\left(F_{1}, \ldots, F_{d}\right)\right)=1$ 그리고 $\kappa_{n}\left(\Pi\left(F_{1}, \ldots, F_{d}\right)\right)=0$;

A2. (Monotonicity) 만약 결합분포 $H_{1}$ 과 $H_{2}$ 가 $H_{1} \prec H_{2}$ 를 만족하면, $\kappa_{d}\left(H_{1}\right) \leq \kappa_{d}\left(H_{2}\right)$;

A3. (Continuity) 만약 결합분포 $\left\{H_{1}, H_{2}, \ldots\right\}$ 이 $H$ 로 pointwise 수렴하면, $\lim _{i \rightarrow \infty} \kappa_{d}\left(H_{i}\right)=\kappa_{d}(H)$;

A4. (Permutation Invariance) $\{1, \ldots, d\}$ 에서 $\{1, \ldots, d\}$ 로의 임의의 permutation, $\rho$ 에 대해서 다음 을 만족한다.

$$
\kappa_{p}\left(X_{1}, X_{2}, \ldots, X_{d}\right)=\kappa_{p}\left(X_{\rho(1)}, X_{\rho(2)}, \ldots, X_{\rho(d)}\right)
$$

A5. (Duality) $\kappa_{d}(\mathbf{X})=\kappa_{p}(-\mathbf{X})$;

A6. (Reflection Symmetry Property) $\sum_{\epsilon_{1}, \ldots, \epsilon_{d}= \pm 1} \kappa\left(\epsilon_{1} X_{1}, \ldots, \epsilon_{d} X_{d}\right)=0$;

A7. (Transition Property) $d \geq 2$ 이면, 다음을 만족하는 임의의 수열 $\left\{r_{1}, r_{2}, \ldots\right\}$ 이 존재한다.

$$
r_{d-1} \kappa_{d-1}\left(X_{2}, \ldots, X_{d}\right)=\kappa_{d}\left(X_{1}, \ldots, X_{d}\right)+\kappa_{d}\left(-X_{1}, X_{2}, \ldots, X_{d}\right)
$$

정리 $3.1 \tau_{d}$ 와 $T_{d}$ 는 일치 측도이다.

증명: 공리 A2를 제외한 공리 A1 A7은 간단한 계산을 통해서 얻어진다. 공리 A2는 Joe (1990)에 증 명되어 있다.

실제 $\tau_{d}$ 와 $T_{d}$ 가 어떠한 차이를 보이는지 다음의 예제를 통해 살펴보도록 하겠다.

예제 3.1: 이번 예제에서는 Farlie-Gumbel-Morgenstern 코풀라 $(d \geq 3)$ 를 고려해본다. 2 차원 FarlieGumbel-Morgenstern 코퓰라는 다음과 같이 정의되고

$$
C\left(u_{1}, u_{2}\right)=u v-\theta\left(1-u_{1}\right)\left(1-u_{2}\right),
$$

이 코퓰라를 다차원으로 확장하는 방법에는 여러 가지가 있지만, 이번 예제에서는 다음과 같이 Kotz 등 (2000)와 Genest 등 (2011)에서 고려된 방법을 사용한다.

$$
C_{\theta}\left(u_{1}, \ldots, u_{d}\right):=\left(\prod_{i=1}^{d} u_{i}\right)\left\{1+\theta \prod_{i=1}^{d}\left(1-u_{i}\right)\right\}, \quad \text { for } \theta \in[-1,1], u_{1}, \ldots, u_{d} \in[0,1],
$$

여기서 $C_{\theta}$ 는 pairwise하게 모두 독립이므로, $C_{\theta}$ 를 코퓰라로 가지는 임의의 랜덤벡터 $\mathbf{X}$ 에 대하여, $T_{d}(\mathbf{X})=0$ 임을 쉽게 보일 수 있다. 반면 Genest 등 (2011)에서 계산된 바와 같이 예를 들어 4 차원인 경우

로 계산할 수 있다.

$$
\tau_{4}(\mathbf{X})=\frac{2 \theta}{567} \neq 0, \quad \text { if } \theta \neq 0
$$


위 예제에서 볼 수 있듯이, $T_{d}$ 는 pairwise한 2 차원 코풀라들의 convex sum으로서, 다차원 변수 간의 관 계를 반영하지 못할 수 있다는 점에 주목해야 한다. 비록 $\tau_{d}$ 와 $T_{d}$ 는 일치 측도이지만, 이 같은 이유로 본 논문에서는 $\tau_{d}$ 를 이용한 분석에 초점을 맞추기로 한다.

\section{Comonotonicity와 $d$-countermonotonicity}

지난 20 년 동안 보험과 금융분야의 여러 학자들은 comonotonicity 개념을 도입해서 발전시켜 왔다. Comonotonicity는 아직도 보험과 금융 분야에서 활발히 연구되고 있는데, 이는 랜덤벡터의 성분이 항상 같은 방향으로 움직이는 것을 의미한다. Comonotonicity에 관련된 자세한 성질은 Dhaene 등 (2002), Cheung (2008), Nam 등 (2011) 등에 잘 정리되어 있고, 수학적으로는 다음과 같이 정의된 다.

정의 4.1 (Dhaene 등, 2002) 랜덤벡터 $\mathbf{X}=\left(X_{1}, \ldots, X_{d}\right)$ 가 임의의 $\mathbf{x}=\left(x_{1}, \ldots, x_{d}\right)$ 에 대해서 다 음을 만족하면

$$
\mathrm{P}\left(X_{1} \leq x_{1}, \ldots, X_{d} \leq x_{d}\right)=\min _{1 \leq i \leq d} \mathrm{P}\left(X_{i} \leq x_{i}\right),
$$

$\mathbf{X}$ 는 comonotonic하다고 한다.

Comonotonic 성질과는 반대 개념으로, countermonotonic 성질을 정의할 수 있다. 2 차원 랜덤벡터가 countermonotonic하다는 것은 두 개의 성분이 항상 다른 방향으로 움직이는 것을 의미하고, 수학적으 로는 다음과 같이 정의된다.

정의 4.2 (Dhaene 등, 2002) 랜덤벡터 $\mathbf{X}=\left(X_{1}, X_{2}\right)$ 가 임의의 $\mathbf{x}=\left(x_{1}, x_{2}\right)$ 에 대해서 다음을 만족 하면

$$
\mathrm{P}\left(X_{1} \leq x_{1}, X_{2} \leq x_{2}\right)=\max \left\{\mathrm{P}\left(X_{1} \leq x_{1}\right)+\mathrm{P}\left(X_{2} \leq x_{2}\right)-1,0\right\},
$$

$\mathbf{X}$ 는 countermonotonic하다고 한다.

3 차원 이상의 랜덤벡터의 성분이 항상 다른 방향으로 움직이는 것은 불가능하므로, Comonotonicity 개 념과는 달리 위에서 정의된 countermonotonicity 개념은 2 차원에 한정된다. 다음의 정리 4.1 은 각각 의 확률함수 $F_{1}$ 과 $F_{2}$ 가 단조증가 할 때 countermotonicity와 동치인 조건을 제시해주는 정리로 Lee와 Ahn (2013)을 요약한 것이다. 증명이 복잡하지 않기 때문에 논문의 완결성을 위해서 증명도 첨부하기 로 한다. 확률함수 $F_{1}$ 과 $F_{2}$ 가 단조증가 한다는 가정은 우리가 관심을 두는 대부분의 확률변수가 만족 하는 강하지 않은 조건이지만, 정리 4.1의 더 일반적인 조건으로의 확장은 Lee와 Ahn (2013)을 참조 하기로 한다. 또한, 이 동치인 조건을 이용하여 정의 4.3 에서는 3 차원 이상의 랜덤백터에서 countermonotonicity의 개념을 정의하였다. 아래 정리의 간결성을 위해 새로운 기호

$$
l_{i}:=\operatorname{ess} \inf \left(X_{i}\right) \quad \text { and } \quad u_{i}:=\operatorname{ess} \sup \left(X_{i}\right), \quad \text { for } i \in\{1, \ldots, d\}
$$

를 정의한다.

정리 4.1 (Lee와 Ahn, 2013) $i=1,2$ 에 대해서 확률함수 $F_{i}(\cdot)$ 가 열린구간 $\left(l_{i}, u_{i}\right)$ 에서 단조 증가하 
는 연속함수라고 가정하면 2 차원 랜덤벡터 $\mathbf{X}=\left(X_{1}, X_{2}\right)$ 가 countermonotonic인 것과 동치인 조건은 다음을 만족하는

$$
f_{1}\left(X_{1}\right)+f_{2}\left(X_{2}\right) \stackrel{\text { a.s. }}{=} 1,
$$

증가함수 $f_{1}$ 과 $f_{2}$ 가 존재하고, 이 함수들이 각각 열린구간 $\left(l_{1}, u_{1}\right)$ 과 $\left(l_{2}, u_{2}\right)$ 에서 단조 증가하는 것이 다.

증명: X가 countermonotonic이라고 가정하면 Dhaene 등 (2002)에 의해 다음의 식

$$
F_{1}\left(X_{1}\right)+F_{2}\left(X_{2}\right) \stackrel{\text { a.s. }}{=} 1
$$

을 쉽게 유도 할 수 있으므로 충분조건이 증명되었다. 이제 필요조건의 증명을 위해 증가함수 $f_{1}$ 과 $f_{2}$ 가 식 $(4.2)$ 를 만족하고, 또한 이 함수들이 각각 열린구간 $\left(l_{1}, u_{1}\right)$ 과 $\left(l_{2}, u_{2}\right)$ 에서 단조증가한다고 가 정하자. 그러면

$$
\left(X_{1}, X_{2}\right) \stackrel{\text { a.s. }}{=}\left(f_{1}^{-1}\left(1-f_{2}\left(X_{2}\right)\right), X_{2}\right)
$$

이고, $f_{1}^{-1}\left(1-f_{2}(\cdot)\right)$ 은 열린구간 $\left(l_{i}, u_{i}\right)$ 에서 감소함수이기 때문에 $\left(X_{1}, X_{2}\right)$ 는, Dhaene 등 $(2002)$ 를 이용해서, comonotonic임을 쉽게 보일 수 있다.

이제 위 정리의 조건을 다음과 같이 정의한다.

\section{조건 $\mathbf{S}$}

$i \in\{1, \ldots, d\}$ 에 대해서, $X_{i}$ 의 확률함수 $F_{i}(\cdot)$ 는 $x \in\left(l_{i}, u_{i}\right)$ 일 때 단조 증가하는 연속함수이다.

다음 정의에서는 조건 $\mathrm{S}$ 를 만족하는 랜덤벡터 $\mathbf{X}$ 에 대해서 $d$ 차원에서의 countermonotonicity 개념을 정의한다.

정의 4.3 (Lee와 $\mathrm{Ahn}, \mathbf{2 0 1 3}$ ) 조건 $\mathrm{S}$ 를 만족하는 확률벡터 $\mathrm{X}$ 에 대해서, 각 열린구간 $\left(l_{i}, u_{i}\right), i=$ $1, \ldots, d$ 에서 단조 증가하는 증가함수 $f_{i}(\cdot), i=1, \ldots, d$ 와 상수 $c \in \mathbb{R}$ 가 존재해서 다음의 식

$$
\sum_{i=1}^{d} f_{i}\left(X_{i}\right) \stackrel{\text { a.s. }}{=} c
$$

을 만족하면 $\mathbf{X}$ 는 $d$-countermonotonic하다고 한다.

논문의 간결성을 위해 위의 정의 4.3 에서는 조건 $\mathrm{S}$ 를 만족하는 확률벡터 $\mathbf{X}$ 에 대해서만 $d$-countermonotonic의 개념을 정의하였다. 좀 더 일반적인 조건에서 $d$ 차원 countermonotonicity 개념의 정의는 $\mathrm{Lee}$ 와 $\mathrm{Ahn}$ (2013)을 참조하기로 한다. 정의 4.3과 정리 4.1로부터 조건 $\mathrm{S}$ 를 만족하는 2 차원 랜덤벡 터 $\mathbf{X}=\left(X_{1}, X_{2}\right)$ 에 대해 2-countermonotonic과 $\mathbf{X}$ 가 countermonotonic 임이 같다는 사실은 자명하다. 다음의 예제를 통해, 가장 간단한 $d$-countermonotonic 형태에 대해서 알아 본다.

예제 4.1: $\mathbf{X}=\left(X_{1}, \ldots, X_{d}\right)$ 가 조건 $\mathrm{S}$ 와 다음을

$$
\sum_{i=1}^{d} X_{i}=c,
$$


만족하는 $d$-countermonotonic 랜덤벡터라고 하자. 식 (4.3)에서 정의된 랜덤벡터 $\mathbf{X}$ 에서, 어느 $X_{j}$ 가 늘어나면 나머지 $X_{i}$ 들, $i \in\{1, \ldots, j-1, j+1, \ldots, d\}$ 의 합이 줄어들게 되므로, 이는 2 차원 countermonotonic 개념의 다차원으로의 자연스러운 확장으로 해석될 수 있다.

정의 4.4 (Lee와 Ahn, 2013) 조건 $\mathrm{S}$ 를 만족하는 랜덤벡터 $\mathbf{X}=\left(X_{1}, \ldots, X_{d}\right)$ 와 자연수 $m(<d)$ 에 대해서 $\left(X_{i_{1}}, \ldots, X_{i_{m}}\right)$ 가 countermonotonic 인 집합 $\left\{i_{1}, \ldots, i_{m}\right\}(\subseteq\{1, \ldots, d\})$ 이 존재하면, $\mathbf{X}$ 를 partially $m$-countermonotonic 하다고 한다.

Partially $m$-countermonotonic의 간단한 예제는 다음에서 살펴볼 수 있다.

예제 4.2: 조건 $\mathrm{S}$ 를 만족하는 랜덤벡터 $\mathbf{X}=\left(X_{1}, X_{2}, X_{3}, X_{4}\right)$ 가 다음의 성질

$$
\mathbf{X} \stackrel{\mathrm{d}}{=}\left(X_{1}, X_{1},-X_{1},-X_{1}\right)
$$

을 만족한다고 하자. $\mathbf{X}$ 의 각 성분의 합이 0 이 되므로 $\mathbf{X}$ 는 4-countermonotonic임을 쉽게 보일 수 있다. 또한, $\left(X_{2}, X_{3}\right)$ 가 2-countermotonic임을 쉽게 보일 수 있고, 따라서 $\mathbf{X}$ 는 partially 2countermonotonic이다.

\section{5. 다차원 켄달의 타우의 최소값}

다차원 켄달의 타우의 해석을 돕기 위해서 가능한 값의 범위에 대한 이해가 필요하다. 식 (3.2)에 정의 된 $\tau_{d}(\cdot)$ 의 정의로부터, $F\left(x_{1}, \ldots, x_{d}\right)=M\left(x_{1}, \ldots, x_{d}\right)$ 이면 $\tau_{d}(\mathbf{X})$ 가 1 이라는 것과 $F\left(x_{1}, \ldots, x_{d}\right)=$ $\Pi\left(x_{1}, \ldots, x_{d}\right)$ 이면 $\tau_{d}(X)=0$ 이라는 것을 공리 $\mathrm{A}$ 와 정리 3.1 을 통해 알고 있다. 하지만, $\tau_{d}(\mathbf{X})$ 의 정의 로부터 그것의 최소값을 유도하는 것은 자명하지 않다. 일반적으로 Scarsini (1984)와 Taylor (2007)에 서 지적되었듯이, 공리 $\mathrm{A}$ 를 만족하는 일치 측도 $\kappa_{d}(\mathbf{X})$ 의 하한에 대해서는 알려져 있지 않을 뿐만 아니 라, $\kappa_{d}(\mathbf{X}) \geq-1$ 인지도 자명하지 않다. 다음의 Proposition 1에서는 다차원 켄달의 타우에 관해서 그 것의 최소값을 제공해준다.

Proposition 1 (Kendall과 Smith, 1940 그리고 Genest 등, 2011) 임의의 랜덤벡터 $\mathbf{X}=\left(X_{1}\right.$, $\left.\ldots, X_{d}\right)$ 는 다음의 부등호

$$
\tau_{d}(\mathbf{X}) \geq-\frac{1}{2^{d-1}-1} \quad \text { 그리고 } \quad T_{d}(\mathbf{X}) \geq-\frac{1}{2\lfloor(d+1) / 2\rfloor-1}
$$

를 만족시킨다.

편의상 위 식 (5.1)의 최소값들을 다음과 같이 정의하자.

$$
L_{1, d}=-\frac{1}{2^{d-1}-1} \quad \text { 그리고 } \quad L_{2, d}=-\frac{1}{2\lfloor(d+1) / 2\rfloor-1} .
$$

Genest 등 (2011)에서 Gumbel-Hougaard 코퓰라의 예를 통해 $\tau_{d}(\mathbf{X})=L_{1, d}$ 를 만족하는 예를 보였고, $T_{d}(\mathbf{X})=L_{2, d}$ 를 만족하는 랜덤벡터 $\mathbf{X}$ 를 찾기 위해 다음의 예제를 살펴보기로 하자.

예제 5.1: [Genest 등, 2011] 랜덤벡터 $\mathbf{X}=\left\{X_{1}, \ldots, X_{d}\right\}$ 를 다음과 같이 정의하자.

$$
X_{i}=\left\{\begin{aligned}
X_{1}, & 1<i \leq\lfloor d / 2\rfloor, \\
-X_{1}, & i>\lfloor d / 2\rfloor .
\end{aligned}\right.
$$


그러면 $\tau\left(X_{1}, X_{1}\right)=1, \tau\left(X_{1},-X_{1}\right)=-1$ 이므로 $T_{d}(\mathbf{X})=-\mathbf{1} /\{\lfloor(\mathbf{d}+\mathbf{1}) / \mathbf{2}\rfloor-\mathbf{1}\}$ 임을 쉽게 보일 수 있 다.

정의 4.2 에서 정의된 2 차원 countermonotonic 벡터의 결합분포의 코퓰라는 코풀라 하한인, $W(\mathbf{u})$ 와 일 치함을 쉽게 알 수 있다 (예를 들어 Dhaene 등 (2002)). 하지만 2절에서 살펴본바와 같이 3차원 이 상의 코퓰라에 대해서는 하한인 코퓰라가 존재하지는 않으므로, countermonotonicity의 정의에 어려 움이 있어, 이에 대한 대체 개념으로 $d$-countermonotonicity를 사용함을 알아보았다. 정리 3.1 로부터 $\tau_{d}(\cdot)$ 가 일치 측도로서 공리 $\mathrm{A} 2$ (monotonicity)를 만족시킴을 알기 때문에 $d$-countermononicity가 2 차 원 countermonotonicity의 자연스러운 확장이 되기 위해서는, 랜덤벡터 $\mathbf{X}$ 가 $d$-countermonotonic일 때

$$
\tau_{d}(\mathbf{X})=L_{1, d}
$$

를 만족하는 것을 기대할 수 있다. 다음은 이러한 $d$-countermononicity와 $\tau_{d}(\cdot)$ 의 하한과의 관계를 제 공해 주는 정리로 Lee와 Ahn (2013)을 요약한 것이다. 증명이 간단하고 또한 논문의 완결성을 위해서 증명도 첨부하기로 한다.

정리 5.1 (Lee와 $\mathbf{A h n , ~ 2 0 1 3 ) ~ ㅈ ㅗ ㄱ ㅓ ㄴ ~} \mathrm{S}$ 를 만족하는 랜덤벡터 $\mathbf{X}=\left(X_{1}, \ldots, X_{d}\right)$ 는 다음을 만족한다.

i. 만약 $\mathbf{X}$ 가 $d$-countermonotonic이면 $\tau_{d}(\mathbf{X})=L_{1, d}$ 이다.

ii. 만약 $\mathbf{X}$ 가 partially $m$-countermonotonic이면 $\tau_{d}(\mathbf{X})=L_{1, d}$ 이다.

증명: 우선 $\mathbf{X}=\left(X_{1}, \ldots, X_{d}\right)$ 가 $d$-countermonotonic이라고 가정하고, $\mathbf{X}^{*}=\left(X_{1}^{*}, \ldots, X_{d}^{*}\right)$ 를 그의 독립인 동일한 분포라고 하자. 위의 첫 번째 명제를 보이기 위해서는, 식 (3.2)에 의해서, 다음을

$$
\mathbb{P}\left(X_{1} \leq X_{1}^{*}, \ldots, X_{d} \leq X_{d}^{*}\right)=0,
$$

보이면 충분하다. 우선 $\mathbf{X}$ 가 $d$-countermonotonic이므로 다음을 만족하는

$$
f_{1}\left(X_{1}\right)+\cdots+f_{d}\left(X_{d}\right) \stackrel{\text { a.s. }}{=} c
$$

증가함수 $f_{1}, f_{2}, \ldots, f_{d}$ 와 상수 $c \in \mathbb{R}$ 를 고려하자. 그러면 우리는 다음의 부등식을 가진다.

$$
\begin{aligned}
\mathbb{P}\left(X_{1} \leq X_{1}^{*}, \ldots, X_{d} \leq X_{d}^{*}\right) & =\mathbb{P}\left(f_{1}\left(X_{1}\right) \leq f_{1}\left(X_{1}^{*}\right), \ldots, f_{d}\left(X_{d}\right) \leq f_{d}\left(X_{d}^{*}\right)\right) \\
& \leq \mathbb{P}\left(\sum_{i=1}^{d-1} f_{i}\left(X_{i}\right) \leq \sum_{i=1}^{d-1} f_{i}\left(X_{i}^{*}\right), f_{d}\left(X_{d}\right) \leq f_{d}\left(X_{d}^{*}\right)\right) \\
& \leq \mathbb{P}\left(c-f_{d}\left(X_{d}\right) \leq c-f_{d}\left(X_{d}^{*}\right), f_{d}\left(X_{d}\right) \leq f_{d}\left(X_{d}^{*}\right)\right) \\
& =0,
\end{aligned}
$$

여기서 마지막 등호는 랜덤벡터 $\mathbf{X}$ 가 연속임으로부터 얻어진다. 이제 식 (5.2)에 의해서

$$
\mathbb{P}\left(X_{1} \leq X_{1}^{*}, \ldots, X_{d} \leq X_{d}^{*}\right)=0
$$

임을 보일 수 있다.

이제 랜덤벡터 $\mathbf{X}=\left(X_{1}, \ldots, X_{d}\right)$ 가 partially $m$-countermonotonic임을 가정하고 $(1<m<d)$, 두 번 
째 명제를 보이겠다. 편의상 $X_{i}$ 들의 순서를 바꾸어서 $\left(X_{1}, X_{2}, \ldots, X_{m}\right)$ 가 $m$-countermonotonic이라 고 가정하여도 무방하고, 첫 번째 명제의 증명과 비슷하게 우리는

$$
\mathbb{P}\left(X_{1} \leq X_{1}^{*}, X_{2} \leq X_{2}^{*}, \ldots, X_{m} \leq X_{m}^{*}\right)=0,
$$

임을 보이면 충분하다. 식 (5.3)은 아래의 식과

$$
\mathbb{P}\left(X_{1} \leq X_{1}^{*}, \ldots, X_{d} \leq X_{d}^{*}\right) \leq \mathbb{P}\left(X_{1} \leq X_{1}^{*}, X_{2} \leq X_{2}^{*}, \ldots, X_{m} \leq X_{m}^{*}\right)
$$

$\left(X_{1}, X_{2}, \ldots, X_{m}\right)$ 가 $m$-countermonotonic이라는 사실로부터 자명하다.

비록 본 논문의 주된 관심은 아니지만 랜덤벡터 $\mathbf{X}=\left(X_{1}, \ldots, X_{d}\right)$ 가 $d$-countermonotonic이거나 partially $m$-countermonotonic일 때, $T_{d}(X)$ 가 하한인, $L_{2, d}$ 를 가질 수 있는 지 궁금하다. 다음의 예제는 이것이 사실이 아님을 보여주는 예제이다.

예제 5.2: 조건 $\mathrm{S}$ 를 만족하는 랜덤벡터 $\mathbf{X}=(3 X,-X,-X,-X)$ 는 $d$-countermonotonic이고 또한 partially 2-countermonotonic임을 쉽게 보일 수 있다. 하지만

$$
T_{4}(\mathbf{X})=0
$$

임을 쉽게 보일 수 있고, 확실히 $0>L_{2,4}=-1 / 3$ 이다. 따라서 $\mathbf{X}$ 는 $d$-countermonotonic인 동시에 partially 2-countermonotonic이지만 $T_{4}(\mathbf{X})$ 의 하한을 얻지 못한다.

위의 정리 5.1 과 예제 5.2 등을 통해 볼 때, $\tau_{d}(\cdot)$ 는 코퓰라 전체의 구조를 반영하는 측도인 반면, $T_{d}(\cdot)$ 는 그러하지 못함을 알 수 있다. 따라서 다차원 문제를 정보의 손실없이 접근하는 방법으로는 $\tau_{d}(\cdot)$ 가 더 적합하다고 판단되므로, 우리는 아래에서 $\tau_{d}(\mathbf{X})$ 에 초점을 맞추어서 그것의 통계학적 추론을 살펴보 고 실제 분석사례 통해 해석을 구체화해보도록 하겠다.

먼저 $\tau_{d}(\mathbf{X})$ 의 통계적 추론에 대해서 알아본다. 이제 다음의 랜덤벡터들이

$$
\mathbf{X}=\left(X_{1}, X_{2}, \ldots, X_{d}\right), \mathbf{X}_{1}=\left(X_{1,1}, X_{1,2}, \ldots, X_{1, d}\right), \ldots, \mathbf{X}_{n}=\left(X_{n, 1}, X_{n, 2}, \ldots, X_{n, d}\right),
$$

i.i.d 관측이고 $F$ 를 분포함수로 가진다고 하자. 우선 $\tau_{d}(\mathbf{X})$ 의 추정량은 다음과 같이 정의된다.

$$
\tau_{d, n}:=\frac{1}{2^{d-1}-1}\left[\frac{2^{d}}{n(n-1)} \sum_{i \neq j} \mathbb{I}\left\{X_{i, 1} \leq X_{j, 1}, X_{i, 2} \leq X_{j, 2}, \ldots, X_{i, d} \leq X_{j, d}\right\}-1\right] .
$$

이제 U-statistic 이론 (van der Vaart, 1998)과, 간단한 계산을 통해 다음의 정리를 쉽게 유도 할 수 있 다.

정리 5.2 (Genest 등, 2011) 만약 $\mathrm{X}_{1}, \mathrm{X}_{2}, \ldots, \mathrm{X}_{n}$ 이 서로 독립인 $d$ 차원 랜덤벡터이고, $F$ 를 분포함 수로 가진다고 하자. 그러면

$$
\sqrt{n}\left(\tau_{2, n}-\tau_{d}\right) \stackrel{\mathrm{d}}{\longrightarrow} \mathrm{N}\left(o, \sigma_{d}^{2}(X)\right)
$$

이다. 여기서 점근적 분산, $\sigma_{d}^{2}(X)$ 는

$$
\sigma_{d}^{2}(X)=\left(\frac{2^{d}}{2^{d-1}-1}\right)^{2} \operatorname{var}\left(F_{d}(X)+\bar{F}_{d}(X)\right)
$$

와 같이 정의된다. 


\section{6. 자료분석}

이 절에서는 실제 자료분석을 통해 다차원 Kendall'a tau에 대한 해석을 좀 더 구체적으로 살펴볼 것이 다. 편의상 다음 예제들에서 사용되는 확률벡터 $\mathbf{X}$ 는 조건 $\mathrm{S}$ 를 만족한다고 가정한다.

예제 6.1: [Bauxite Example] 다차원 켄달의 타우의 첫 번째 응용사례로 알루미늄의 원광인 보크사이 트(Bauxite)를 구성하는 여러 광물 가운데 대표적인 3가지 광물 Albite, Blandite, Cornite를 살펴보기 로 한다. 실제 실험을 통해 25 개 암석의 광물구성성분을 조사하였고, 각 광물이 차지하고 있는 중량의 백분율 값이 주어져 있다 (Aitchison, 1986). 편의상 위의 3 가지 광물을 각각 $\mathrm{A}, \mathrm{B}, \mathrm{C}$ 라고 부르도록 하 겠다. 이 3 차원 변수에 대해 식 (5.4)를 이용하면 켄달의 타우 값의 추정량, $\tau_{3, n}=-0.2933$ 을 얻을 수 있고, 정리 5.2 를 이용하여 구한 신뢰구간은 $(-1 / 3,-0.2425)$ 이다. 여기서 자료의 숫자 $(n=25)$ 가 크 지 않으므로 부츠트랩(Bootstrap) 방법을 사용하여 신뢰구간, $(-1 / 3,-0.2267)$ 을 구해보았고, 이는 정 리 5.2 를 사용한 신뢰구간과 유사하였다. 3 차원의 경우, 켄달의 타우의 하한은 $-1 / 3$ 으로 주어지는 것 을 앞의 절에서 밝혔는데, 관측된 통계량의 값이 하한에 가까우므로, 하한을 얻게 되는 충분조건인 3 counter monotonic이 만족하는 지의 여부를 확인해보는 것은 의미가 있다고 할 수 있겠다. 이를 살펴 보기 위해 $\mathrm{A}, \mathrm{B}, \mathrm{C}$ 의 합을 구한 후 그려보았다. Figure 6.1(a)에서 보이는 것처럼 약간의 변동성은 있 지만 대체로 합은 상수에 가까웠다. 따라서 랜덤벡터 $(\mathrm{A}, \mathrm{B}, \mathrm{C})$ 는 어느 한 성분이 많아지면 다른 성분 들이 차지하는 비중이 줄어드는 음의 함수관계, 즉 3-countermonotonic 성질을 가짐을 알 수 있다. 또 한, 이는 이 세가지 광물을 보크사이트를 구성하는 주요성분 (우리의 예제에서는 대략 $80 \%$ )으로 이해할 때 얻어지는 당연한 결과이기도 하다.

예제 6.2: [Statistician Example] 두 번째 응용사례로 학술활동을 하는 한 통계학자의 활동 자료 Aitchison (1986)를 분석해보았다. 분석대상 변수는 교육 $\left(X_{1}=\right.$ teaching), 상담 $\left(X_{2}=\right.$ consultation), 행정 ( $X_{3}=$ administration) 3 개이며, 각 변수는 하루 24 시간 중에 해당하는 활동에 투자하 는 시간의 비율을 값으로 갖고있다. 이 자료는 이월효과(carry-over effect)를 피하기 위해 격주로 하 루를 랜덤하게 뽑은 후 일과를 기록한 것이며 총 관측 수는 20 개이다. 위의 예제 6.1 과 같이, 이 랜덤 벡터 $\mathbf{X}=\left(\mathbf{X}_{\mathbf{1}}, \mathbf{X}_{\mathbf{2}}, \mathbf{X}_{\mathbf{3}}\right)$ 에 대한 켄달의 타우 값의 추정량, $\tau_{3, n}=-0.2140$ 을 계산할 수 있고, 이의 $95 \%$ 신뢰구간은 $(-0.3017,-0.1264)$ 이었다. 부츠트랩(Bootstrap) 방법을 사용하여 구한 신뢰구간은 $(-0.3053,-0.1298)$ 으로 정리 5.2 를 사용한 신뢰구간과 거의 유사하였다. 앞의 사례와 마찬가지로 관측 된 통계량의 값이 이론적인 하한에 가까우므로 3-counter monotonic에 대한 조건이 만족하는 지 살펴 보는것이 의미가 있다고 보여진다. 이를 위해 다음과 같이 단조 증가 함수 $f_{1}, f_{2}, f_{3}$ 를 정의 하면

$$
f_{1}(x)=\frac{x^{0.2}}{15}, \quad f_{2}(x)=\frac{x^{0.2}}{5}, \quad f_{3}(x)=\frac{x^{0.2}}{5} .
$$

이들의 합, $f_{1}\left(X_{1}\right)+f_{2}\left(X_{2}\right)+f_{3}\left(X_{3}\right)$ 은 Figure 6.1(b)에서 보이는 바와 같이 대략 $c=0.3$ 에 근사한다. 따라서 랜덤벡터 $\mathbf{X}$ 에 대해서 3-counter monotonic 조건이 잘 만족되고 있음을 알 수 있다. 실제 이 자 료는 24 시간 중의 해당 활동에 투자하는 시간의 비율이므로, 여가 및 잠에 투자되는 시간을 모두 고려한 다면 전체의 합은 반드시 1 이 될 것이다. 여가 및 잠 자는 시간에 큰 변동이 없는한 우리가 고려한 세 변 수 사이에는 음의 함수관계가 있을 것으로 예상되므로 랜덤벡터 $\mathbf{X}$ 에 대한 3-countermonotonic이 성립 함은 쉽게 유추할 수 있는 결과이기도 하다.

예제 6.3: [Population Example] 마지막 응용사례로 미국의 동부, 중부, 서부의 대표적인 도시들인 Boston, Chicago, Los Angeles의 인구가 당시 미국 전체 인구에서 차지하는 비율을 1900 년부터 1990년 

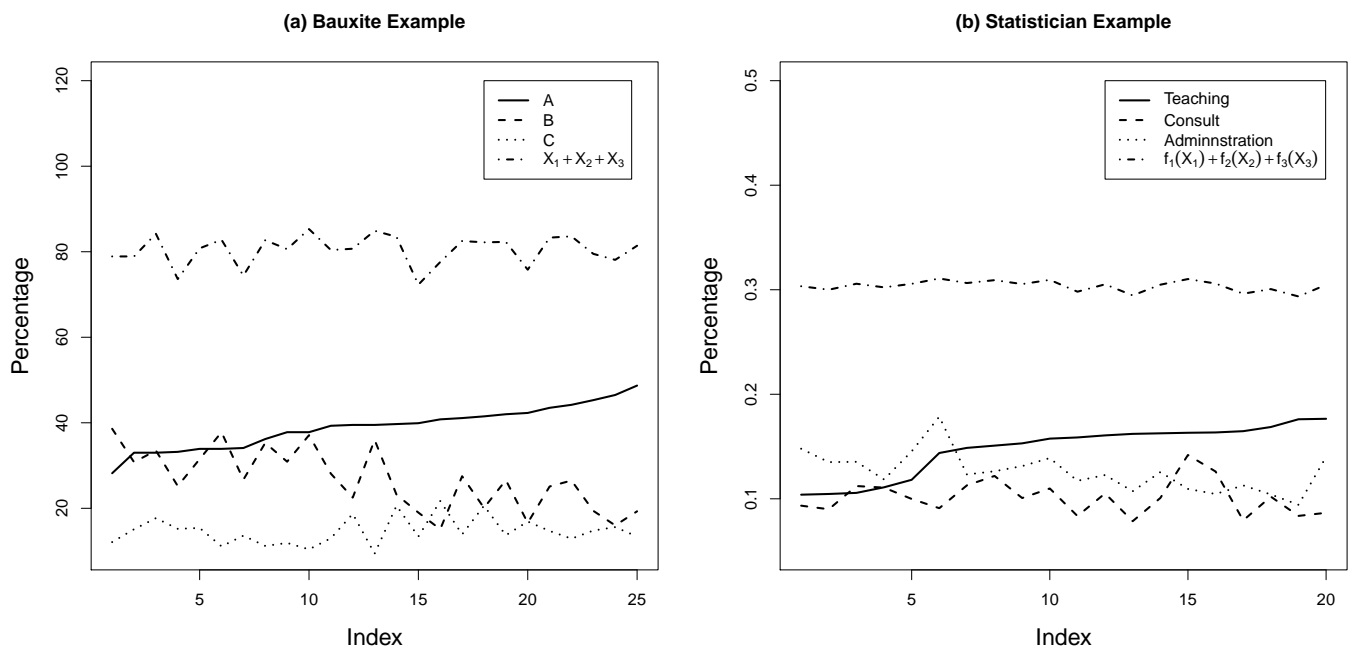

Figure 6.1. Composition of minerals in the Bauxite example(a), and composition of statistican's activity in the statistician example(b). (For convenience, indices are sored by A and Teaching, respectively, in each figures.)

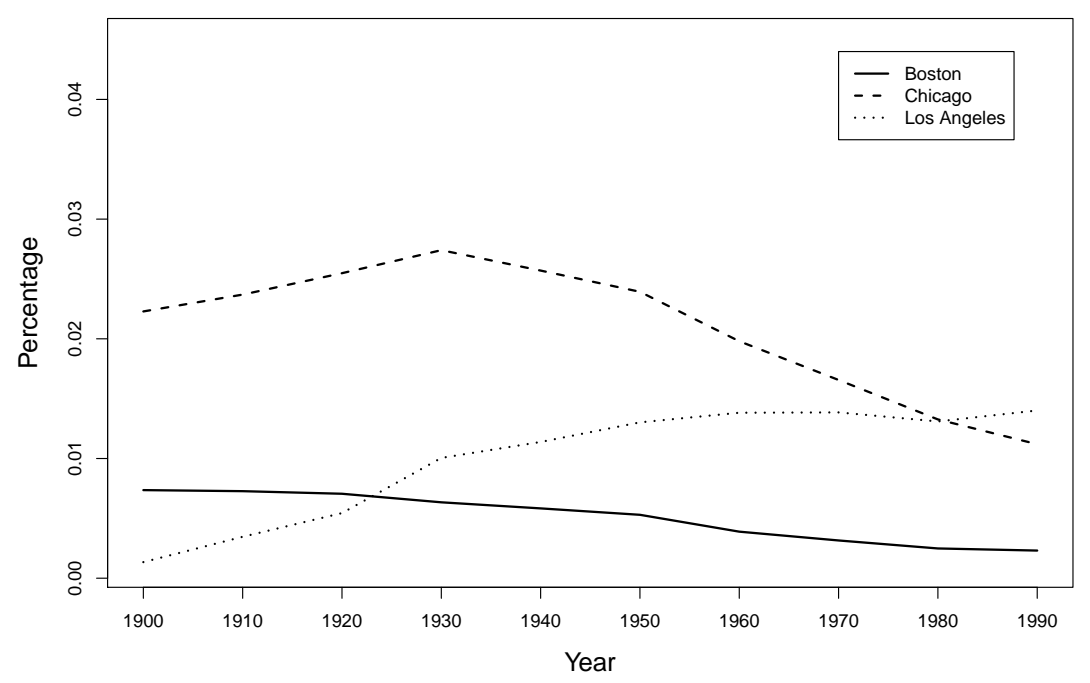

Figure 6.2. Composition of population in Boston, Chicago and Los Angeles in the population example.

까지 10년마다 조사한 자료를 살펴보자. 위 자료는 http://physics.bu.edu/ redner/에서 쉽게 얻을 수 있다. 다음의 Figure 6.2 는 이 시기에 세 도시의 인구 비율을 나타낸 그래프로, 이시기에 있었던 미국의 인구이동을 보여주는 좋은 예이다. 동부의 대표적인 도시인 Boston의 인구비율이 계속 감소하고 있는 반면, 서부의 대표적인 도시인 Los Angeles의 인구비율은 증가하고 있고, 또한 중부에 있는 Chicago의 인구비율은 1930 년까지 증가세를 보이다가 이후 감소세에 있는 것을 알 수 있다. 이는 미국의 급격한 인구팽창과 교통 단가의 하락 그리고 골드러쉬등의 이유로 19 세기부터 시작돼 20 세기에도 계속 되었던 동부에서 서부로의 인구이동 현상을 잘 보여 주고 있는 예제로, 정의 4.3 를 만족하는 간단한 형식의 증 
가함수 $f_{1}(\cdot), f_{2}(\cdot), f_{3}(\cdot)$ 를 찾는 것은 어렵지만, 이 세도시의 인구비율은 3 -countermonotonic과 연관된 것으로 추측해볼 수 있다. 따라서 위 세변수의 켄달의 타우가 하한인 $-1 / 3$ 에 가까울 것으로 추측할 수 있고, 정리 5.1 에 의해 실제로 위 3 차원 변수에 대한 켄달의 타우 값의 추정량, $\tau_{3, n}=-0.2741$ 을 얻 을 수 있고, 이의 $95 \%$ 신뢰구간은 $(-1 / 3,-0.1456)$ 이었다. 부츠트랩(Bootstrap) 방법을 사용하여 구 한 신뢰구간은 $(-1 / 3,-0.1556)$ 으로 정리 5.2 를 사용한 신뢰구간과 유사하였다.

\section{7. 결론}

본 논문에서는 다차원으로의 켄달의 타우의 확장과 의미에 대해 살펴보았다. 이변량의 경우, 임의의 코 퓰라의 하한에 대해 잘 이해하고 있고 이 하한에서 켄달의 타우의 최소값이 나타나기 때문에 켄달의 타 우가 음수일 때 변수들 간의 관계를 해석하는 것이 어렵지 않다. 그러나, 3 차원 이상의 경우 프레쳇호프딩의 하한이 코퓰라가 아니므로 켄달의 타우의 최소값이 어떠한 특성을 가진 코퓰라에서 발생하는 지에 대한 논의는 별개로 다루어져야 하며, 본 논문에서는 실제 자료에서 자주 관측되는 한 조건인 $d-$ countermonotonicty에 대해 정리해 보았다. 이 개념이 변수들 사이의 관계를 이해하는 데 도움이 된다 는 사실을 몇 가지 실제 사례를 통해 살펴보았다. 하지만, 정리 5.1은 켄달의 타우가 최소값을 갖는 충 분조건에 대해서만 논의할 뿐만 아니라, 대상이 켄달의 타우, $\tau_{d}$ 에 한정되어 있기 때문에, 앞으로 다차 원 일치 측도가 최소값을 갖기 위한 조건을 구체화할 수 있는 연구가 필요할 것으로 본다. 한편, 본 연구 의 세 번째 예제에서 보여진 것처럼 $d$-countermonotonicty를 확인해 볼 수 있는 간단한 증가함수를 찾 기 어려울 때도 있다. 이러한 경우 비모수 함수 추정기법을 통해 이 문제를 체계적으로 다룰 수 있는 통 계적인 방법에 관한 연구가 필요하다. 또한, 다양한 조건하에서 대표본 근사를 통해 얻어진 이론적인 신 뢰구간과 부츠트랩으로 얻어진 신뢰구간의 성능이 소표본에서 어느 정도 믿을만한가에 대해 면밀히 검 토해보는 것 또한 앞으로의 중요한 연구로 사료된다.

\section{References}

Aitchison, J. (1986). The Statistical Analysis of Compositional Data, Chapman and Hall, New York.

Cherubini, U., Luciano, E. and Vecchiato, W. (2004). Copula Methods in Finance, Wiley Finance Series, John Wiley \& Sons Ltd.

Cheung, K. C. (2008). Characterization of comonotonicity using convex order, Insurance: Mathematics \& Economics, 43, 403-406.

Dhaene, J., Denuit, M., Goovaerts, M. J., Kaas, R. and Vyncke, D. (2002). The concept of comonotonicity in actuarial science and finance: theory, Insurance: Mathematics \& Economics, 31, 3-33.

Genest, C., Nešlehová, J. and Ben Ghorbal, N. (2011). Estimators based on Kendall's tau in multivariate copula models, Australian \& New Zealand Journal of Statistics, 53, 157-177.

Joe, H. (1990). Multivariate concordance, Journal of Multivariate Analysis, 35, 12-30.

Kendall, M. G. and Smith, B. B. (1940). On the method of paired comparisons, Biometrika, 31, 324-345.

Kotz, S., Balakrishnan, N. and Johnson, N. L. (2000). Continuous Multivariate Distributions, Models and Applications, Wiley-Interscience.

Lee, W. and Ahn, J. Y. (2013). On the Multivariate Extension of Countermonotonicity and its Application to Measures of Concordance, Manuscript for Publication.

Nam, H. S., Tang, Q. and Yang, F. (2011). Characterization of upper comonotonicity via tail convex order, Insurance: Mathematics \& Economics, 48, 368-373.

Nelson, R. B. (2006). An Introduction to Copulas, Springer Series in Statistics, Springer, New York.

Scarsini, M. (1984). On measures of concordance, Stochastica, 8, 201-218.

Schmid, F. and Schmidt, R. (2007). Multivariate extensions of Spearman's rho and related statistics, Statistics \& Probability Letters, 77, 407-416. 
Sklar, M. (1959). Fonctions de répartition à $n$ dimensions et leurs marges, Institut de Statistiques de l'Université de Paris, 8, 229-231.

Taylor, M. D. (2007). Multivariate measures of concordance, Annals of the Institute of Statistical Mathematics, 59, 789-806.

van der Vaart, A. W. (1998). Asymptotic Statistics, Cambridge Series in Statistical and Probabilistic Mathematics, 3, Cambridge University Press, Cambridge. 


\title{
다차원 캔달의 타우의 통계학적 응용과 그의 해석
}

\author{
이우주 ${ }^{a} \cdot$ 안재윤 $^{b, 1}$ \\ ${ }^{a}$ 인하대학교 통계학과, ${ }^{b}$ 이화여자대학교 통계학과
}

(2013년 4월 2일 접수, 2013년 4월 28일 수정, 2013년 5월 15일 채택)

\section{요 약}

본 논문에서는 캔달의 타우(Kendall's tau)의 다차원으로의 확장과 그의 통계적 추론 및 해석에 대해 알아본다. 특 히 다차원 캔달의 타우가 음의 값을 가질 때 의미를 해석하기 위해, 그것의 하한이 얻어지는 경우를 직관적으로 이해 할 수 있도록 변수들간의 관계의 관점에서 설명하여본다. 또한 다차원 캔달의 타우를 실제 사례에 적용해 본후, 최 근 Lee와 Ahn에서 연구된 d-countermonotonicity와 partially m-countermonotonic와 같은 새로운 개념을 통하 여 캔달의 타우가 음의 값이 가질 때의 의미에 대해서 논의한다.

주요용어: 코퓰라, 캔달의 타우, comonotonicity, 프레쳇-호프팅 하한.

이 논문은 인하대학교의 지원을 받아 수행되었음 (INHA-44770-01)

1 교신저자: (120-750) 서울시 서대문구 대현동 11-1, 이화여자대학교 통계학과, 조교수.

E-mail: jaeyahn@ewha.ac.kr 